\title{
EDITORIAL
}

\section{Beyond the bins: interpreting and discussing pharmacogenomic reports with psychiatric patients}

\author{
Boadie W. Dunlop iD \\ Department of Psychiatry and Behavioral Sciences, Emory University School of Medicine, Atlanta, GA, USA.
}

\section{Introduction}

With the burgeoning of genetics knowledge in psychiatry, several companies now market pharmacogenomic (PGX) decision support tools (DSTs) to help inform medication selection and dosing for treating depression. A recent meta-analysis of five PGx DSTs tested in randomized controlled trials found a significantly higher remission rate (odds ratio: 1.7, 95\% confidence interval: 1.2-2.5) among patients who received PGX-informed treatment compared to those receiving treatment as usual. ${ }^{1}$ The utility of the PGx tests appears to be greater in patients who have failed to tolerate or respond to at least one prior medication trial. ${ }^{1,2}$ However, recent statements from the US Food and Drug Administration and a position paper from the American Psychiatric Association have raised concerns about whether and in what circumstances clinicians should order PGx testing. ${ }^{3}$ Debate about the role that $P G x$ DSTs should play in psychiatric clinical care is likely to continue in the foreseeable future.

Given this state of the field, it is unsurprising that clinicians may feel uncertain about ordering or interpreting PGx DSTs. It should be noted that the Clinical Pharmacogenetics Implementation Consortium (CPIC) publishes publicly available guidelines based on a consensus of expert opinion. ${ }^{4}$ Among the psychiatric drugs, CPIC assigns the highest level of evidence only for associations between CYP2D6 and CYP2C19 genetic variants and the dosing of tricyclic and selective serotonin reuptake inhibitor antidepressants and atomoxetine. Here, for clinicians who choose to order PGX DSTs, I suggest a structured approach to using these reports.

\section{Genes included in PGx DST reports}

Pharmacokinetic genes are those that produce proteins involved in the metabolism of drugs, including phase I (cytochrome P450 enzymes) and phase II metabolism (e.g., transferases such as UGT1A1 and UGT2B15). For phase I metabolism there are six CYP450 genes typically included in psychiatric PGX DSTs (CYP 1A2, 2B6, 2C9, $2 C 19,2 D 6$, and $3 A 4 / 5)$. Each person has two copies of each of these genes, which are classified into "star" alleles that assign a functional status to the level of

Correspondence: Department of Psychiatry and Behavioral Sciences, Emory University School of Medicine, 12 Executive Park Drive NE, 3rd floor, 30329, Atlanta, GA, USA.

E-mail: bdunlop@emory.edu

Submitted Sep 06 2019, accepted Sep 09 2019, Epub Jan 242020. activity of the enzyme the gene codes for, including increased function, normal function, decreased function and no function. There are also categories for unknown and uncertain function. The specific combination of a person's two star alleles for each CYP gene determines their metabolic phenotype for that CYP enzyme, classified as: ultra-rapid, rapid, normal (previously known as "extensive"), intermediate, or poor metabolizer. ${ }^{5}$ Clinically, the primary concern for ultra-rapid metabolizers is that they will not respond to treatment, whereas for poor metabolizers the concerns are for side effects or other safety issues.

Pharmacodynamic genes are those that code for proteins that are biological targets of a medication, such as monoamine receptors (e.g., HTR2A for the serotonin type 2 receptor) and transporters (e.g., SLC6A4 for the serotonin transporter). To date, the level of evidence that pharmacodynamic genes impact response to antidepressants is generally weaker than that of the pharmacokinetic genes; therefore, there is less consistency across the pharmacodynamic genes that PGx tests include in their DSTs. Some PGx DSTs incorporate Phase III metabolism genes such as $A B C B 1$ and $A B C C 1$ that code for ATP-binding cassette transporters, which actively pump out medications from the central nervous system across the blood brain barrier. PGx reports may also include results for major histocompatibility complex genes, such as $H L A-B^{\star} 1502$, which can represent a major risk factor for Stevens-Johnson syndrome or toxic epidermal necrolysis with carbamazepine or oxcarbazepine.

\section{Discussing PGx results with patients}

An overarching goal in discussing PGx reports is for the patient to understand that the test is not a definitive answer to what will "work," but rather that the test can help reduce uncertainty in selecting medications. For simplicity, the first page of most PGX DST reports presents medication recommendations in color-coded bins. Although these colorful bins represent a report's "bottomline," clinicians can benefit from beginning at the summary of the genetic results before discussing the binned medication recommendations.

How to cite this article: Dunlop BW. Beyond the bins: interpreting and discussing pharmacogenomic reports with psychiatric patients. Braz J Psychiatry. 2020;42:111-112. http://dx.doi.org/10.1590/15164446-2019-0697 
Table 1 Process for discussing pharmacogenomic decision support tools with patients

\begin{tabular}{lll}
\hline Step & Goal & Approach \\
\hline 1 & Prevent misconceptions. Frame expectations. & $\begin{array}{l}\text { Explain that the test only evaluates whether there are gene variants that } \\
\text { may impact a patient's response to medications, and that the test does not }\end{array}$ \\
& $\begin{array}{l}\text { address: 1) heredity of psychiatric disease; } 2) \text { prognosis about the long- } \\
\text { term course of illness; or 3) information about risk for other diseases, such } \\
\text { as Alzheimer's disease. }\end{array}$
\end{tabular}

2 Understand that responses to medications stem from many factors besides genetics.

Understand there is disagreement about the importance of some genes' impact on medication response.

$4 \quad$ Understand the meaning of metabolizer categories of CYP enzymes, especially "Ultra-rapid" versus "Poor."

$5 \quad$ Understand that drugs can directly or indirectly act on transporters and receptors involved in chemical signaling in the brain.

6 Review the medication bin categories and the medication options the clinician is considering based on the test report.

Confirm understanding of how the test is being used in this patient's case.
Explicitly state the non-genetic (i.e., clinical) factors that also inform the physician's medication selection. State that a medication that is helping will not be stopped simply because it appears in a "warning" or "cautionary" use bin.

State that not all the factors that contribute to a patient's ability to respond to or tolerate specific medicines are known.

Discuss pharmacokinetic gene results.

Discuss pharmacodynamic gene results.

Discuss the guidance provided by the medication bins of the decision support tool, framing the discussion around genetic results being only one component of medication selection.

Elicit questions and address concerns.
Table 1 presents a stepwise approach to discussing PGx DSTs. Steps 1 and 2 are best conducted prior to deciding to order the test. Key messages that patients should take away after discussing DST results are that medications listed under a "warning" or "caution" bin can still be appropriate to use, and that medication choice, dosing, and response depend on several factors other than genetics. Additionally, clinicians should be mindful that medication recommendations for the same individual can vary substantially between the different PGx testing companies due to a variety of reasons, so that the tests cannot be considered fully interchangeable. ${ }^{6}$

A potential downside of $P G x$ testing is that patients and clinicians may latch on to a "biological cause" for the lack of improvement. It is important to emphasize that response to treatment also depends on one's psychological and social state and on other medical factors, and that these will need to remain part of the discussion as the patient moves toward wellness. Another risk is that a patient who is doing well on a medication may find that a PGx DST lists their medication in a "caution" bin, and so asks if they should stop it. In this situation, the clinician should educate the patient about the limitations of pharmacogenomic testing and reassure the patient to remain on the effective medication.

\section{Conclusion}

PGx DSTs should not replace clinician judgment in medication selection, which requires consideration of comorbid medical and psychiatric disorders, response and tolerability of prior medication trials, concomitant medications, financial concerns, and the likely level of adherence to the medication. A structured approach to reviewing PGx DST reports supports shared decision-making with the patient and can foster a broader awareness of factors impacting treatment response.

\section{Disclosure}

BWD has received honoraria for consultation work with Myriad Neurosciences (manufacturer of the GeneSight Psychotropic test) and Aptinyx. He has received research support from Acadia, Intra-Cellular Therapies, Takeda, and the National Institutes of Health.

\section{References}

1 Bousman CA, Arandjelovic K, Serafino G, Mancuso SG, Eyre HA, Dunlop BW. Pharmacogenetic tests and depressive symptom remission: a meta-analysis of randomized controlled trials. Pharmacogenomics. 2019;20:37-47.

2 Greden JF, Parikh SV, Rothschild AJ, Thase ME, Dunlop BW, DeBattista C, et al. Impact of pharmacogenomics on clinical outcomes in major depressive disorder in the GUIDED trial: a large, patient- and rater-blinded, randomized, controlled study. J Psychiatr Res. 2019;111:59-67.

3 Zeier Z, Carpenter LL, Kalin NH, Rodriguez Cl, McDonald WM, Widge AS, et al. Clinical implementation of pharmacogenetic decision support tools for antidepressant drug prescribing. Am J Psychiatry. 2018; $175: 873-86$.

4 Clinical Pharmacogenetics Implementation Consortium [Internet]. [cited Nov 07 2019]. https://cpicpgx.org

5 Caudle KE, Dunnenberger HM, Freimuth RR, Peterson JF, Burlison JD, Whirl-Carrillo $\mathrm{M}$, et al. Standardizing terms for clinical pharmacogenetic test results: consensus terms from the Clinical Pharmacogenetics Implementation Consortium (CPIC). Genet Med. 2017; 19:215-23.

6 Bousman CA, Dunlop BW. Genotype, phenotype, and medication recommendation agreement among commercial pharmacogeneticbased decision support tools. Pharmacogenomics J. 2018;18:613-22. 